\section{About the Materials Research Society}

The Materials Research Society (MRS), a not-forprofit scientific association founded in 1973, promotes interdisciplinary goal-oriented basic research on materials of technological importance. Membership in the Society includes almost 16,000 scientists, engineers, and research managers from industrial, government, and university research laboratories in the United States and close to 70 countries.

The Society's interdisciplinary approach differs from that of single-discipline professional societies because it promotes information exchange across the many technical fields touching materials development. MRS sponsors two major international annual meetings encompassing approximately 90 topical symposia, and also sponsors numerous single-topic scientific meetings. The Society recognizes professional and technical excellence and fosters technical interaction in local geographic regions through Sections and University Chapters.

MRS participates in the international arena of materials research through the International Union of Materials Research Societies (IUMRS). MRS is a member of ASTRA and is an affiliate of the American Institute of Physics.

MRS publishes symposium proceedings, $M R S$ Bulletin, Journal of Materials Research, and other publications related to current research activities.

MRS Bulletin (ISSN: 0883-7694, print; ISSN 19381425 , online) is published monthly by the Materials Research Society, 506 Keystone Drive, Warrendale, PA 15086-7573. Copyright (C2010, Materials Research Society. Permission required to reproduce content. Periodical postage paid at Warrendale, PA, and at additional mailing offices. POSTMASTER: Send address changes to MRS Bulletin in care of the Materials Research Society, at the address listed; tel. 724-779-3003; fax 724-779-8313. Printed in the U.S.A. Membership in MRS is \$115 annually for regular members, $\$ 30$ for students. Dues include an allocation of \$29 (\$17 for students) to a subscription to MRS Bulletin. Individual member subscriptions are for personal use only. Non-member subscription rates are $\$ 300$ for one calendar year (12 issues) within the U.S.A. and $\$ 360$ elsewhere. Single copies may be purchased for $\$ 55$ each. Requests from subscribers for missing journal issues will be honored without charge only if received within six months of the issue's actual date of publication; otherwise, the issue may be purchased at the single-copy price. MRS Bulletin is included in Current Contents ${ }^{\circledR}$ Enoineering Computing and Technology: Current Contents ${ }^{\circledast}$ Physical, Chemical, and Earth Sciences, the SciSearch $^{\circledR}$ online database, Research Alert ${ }^{\circledR}$, Science Citation Index ${ }^{\circledR}$, and the Materials Science Citation Index ${ }^{\mathrm{TM}}$. Back volumes of MRS Bulletin are available on microfiche through University Microfilms Inc., 300 North Zeeb Road, Ann Arbor, MI 48106, USA.

Send Letters to the Editor to:

MRS Bulletin

Materials Research Society 506 Keystone Drive

Warrendale, PA 15086-7573 USA

Fax 724-779-8313

Bulletin@mrs.org

Letters must include your name, affiliation, and full contact information.
Editorial Office • 506 Keystone Drive • Warrendale, PA 15086-7573 USA

Tel. 724-779-2747; fax 724-779-8313; Bulletin@mrs.org; http://www.mrs.org/
Editor

E.L. Fleischer

Managing Editor

J. Meiksin

Technical Editor

L. Wilson

Associate Technical Editor

T. Palucka

Editorial Assistant

B. Moriarty

\section{EDITORIAL BOARD}

P.S. Drzaic, Chair

Drzaic Consulting Services

Morgan Hill, CA

V.S. Arunachalam

Center for Study of Science,

Technology \& Policy

Bangalore, India

M.-I. Baraton

University of Limoges

Limoges, France

R.C. Cammarata

Johns Hopkins University

Baltimore, MD

\section{VOLUME ORGANIZERS}

\section{9}

A. Misra

Los Alamos National Laboratory

2010

T.J. Balk

University of Kentucky

2011

K.-S. Choi

Purdue University

2012

L. Jiang

Chinese Academy of Sciences

\section{Guest Editors}

C. Eberl and T. Saif

Special Contributors

R. Armstrong, B.V.R. Chowdari,

S. Cooper, K.P. Herlihy, G. Kiriakidis,

J. Li, C. McCormick, M. Meyers,

G.R. Rao, S. Trohalaki, and T.D. Ziebell

Special Consultant

A. Sellinger

\section{InterFaces}

C. Trager-Cowan (Chair), Y.-T. Cheng,

A. Misra, and U. Ramamurty
MRS Office of Public Affairs

R. Kelley

499 South Capitol Street SW,

Suite 600, Washington, DC 20003

Art Director

K. Bruniany

Production/Design

Franklin Design and SPI Technologies, Inc.

Advertising

M.E. Kaufold and D.L. Watterson

Circulation

M. Judt

\section{Fornaro}

University of Uruguay

Montevideo, Uruguay

H.-U. Habermeier

Max Planck Institute

Stuttgart, Germany

S.C. Moss

Aerospace Corp.

Los Angeles, CA

J.A. Nucci

Cornell University

Ithaca, NY

L.J. Olafsen

Baylor University

Waco, TX
D.N. Seidman

Northwestern University

Evanston, IL

C. Trager-Cowan

University of Strathclyde

Glasgow, UK

J.R. Weertman

Northwestern University

Evanston, IL

E. Werwa

Washington, DC

S.M. Yalisove

University of Michigan

Ann Arbor, MI

\begin{tabular}{lll}
\hline & 2010 MRS OFFICERS & \\
\hline President & Secretary & Immediate Past President \\
$\begin{array}{l}\text { D.S. Ginley } \\
\text { National Renewable Energy Laboratory }\end{array}$ & B.J.H. Stadler & S.P. Baker \\
& University of Minnesota & Cornell University \\
Vice President and President-Elect & Treasurer & Executive Director \\
J.J. De Yoreo & M. Fitzsimmons & Materials Research Society \\
Lawrence Berkeley National Laboratory & Los Alamos National Laboratory & T. Osman
\end{tabular}

R. O'Hayre

Colorado School of Mines

D. Cahen

Weizmann Institute of

Science

R.T. Collins

Colorado School of Mines

S. Kalinin

Shull

Northwestern University

S. Stemmer

University of California,

Santa Barbara

S. Streiffer

Argonne National

Laboratory

G. Redmond

University College Dublin

S.E. Shaheen

University of Denver

Moss

S. Moss

Aerospace Corp.

\section{Materials Research Society}

$\begin{array}{ll}\text { President } & \text { First Vice President } \\ \text { H. Katz } & \text { B.V.R. Chowdari } \\ \text { Johns Hopkins University } & \text { Natl. Univ. of Singapore } \\ \text { Baltimore, MD, USA } & \text { Singapore }\end{array}$

IUMRS ADHERING BODIES

Australian Materials Research Society (A-MRS)

J.S. Williams, Australian National University

Brazil Materials Research Society (B-MRS)

F. Lázaro Freire Jr., PUC-Rio

Chinese Materials Research Society (C-MRS)

X. Peng, Beijing, China

European Materials Research Society (E-MRS)

F. Priolo, INFM

Materials Research Society (MRS)

D.S. Ginley, National Renewable Energy Laboratory

\title{
INTERNATIONAL UNION OF MATERIALS RESEARCH SOCIETIES
}

$\begin{array}{lll}\text { Second Vice President } & \text { Secretary } & \text { Treasurer } \\ \text { O. Takai } & \text { Q. Feng } & \text { Y. Han } \\ \text { Nagoya University } & \text { University of Science } & \text { Inst. of Aeronautical } \\ \text { Japan } & \text { and Technology } & \text { Materials } \\ & \text { Beijing, China } & \text { Beijing, China }\end{array}$

mmediate Past President G.M. Crean

Atomic Energy Commission Grenoble, France Materials Research Society of Singapore (MRS-S) B.V.R. Chowdari, National University of Singapore

Materials Research Society of Argentina (MRS-Argentina) J. Ovejero García, CNEA

Materials Research Society of India (MRS-I)

T. Ramasami, Ministry of Science and Technology

Materials Research Society of Japan (MRS-J)

N. Kishimoto, National Institute for Materials Science

Materials Research Society of Korea (MRS-K)

J.-W. Park, Hanyang University

Materials Research Society of Russia (MRS-Russia)

N.Z. Lyakhov, Russian Academy of Sciences
General Secretary

R.P.H. Chang

Northwestern University

Evanston, IL, USA 